\title{
25 Research Soure \\ The Benefits of Guilu Erxian Jiao for Patients With Osteoporosis: A Retrospective Study
}

\section{Yuan-Xin Lai ( $\nabla$ u9802401@cmu.edu.tw )}

Chang Gung Memorial Hospital https://orcid.org/0000-0001-7305-0496

\section{Chu-Yao Tseng}

Chang Gung Memorial Hospital Taoyuan Branch: Taoyuan Chang Gung Memorial Hospital

\section{Huang-Yu Yang}

Chang Gung Kidney Research Center

\section{Sien-Hung Yang}

Chang Gung Memorial Hospital Taipei Branch: Chang Gung Memorial Hospital

\section{Pai-Wei Lee}

Chang Gung Memorial Hospital Linkou Main Branch: Chang Gung Memorial Hospital

\section{Tsung-Hsien Yang}

New Taipei City Municipal Tucheng Hospital

\section{Research}

Keywords: Chinese herbal medicine, Guilu Erxian Jiao, osteoporosis, T-score, fracture

Posted Date: August 6th, 2021

DOI: https://doi.org/10.21203/rs.3.rs-764315/v1

License: (c) (1) This work is licensed under a Creative Commons Attribution 4.0 International License. Read Full License 


\section{Abstract}

Objective: The objective of this study was to evaluate whether the Chinese herbal medicine formula Guilu Erxian Jiao can improve the T-score in osteoporosis patients and even decrease the fracture rate.

Design: This is a retrospective study.

Subjects and Setting: Osteoporosis participants were selected from the Chang Gung Memorial Hospital (CGMH) database from 2000 to 2019.

Interventions: The patients were administered Guilu Erxian Jiao pills.

Outcome measures: We analyzed the change of T-score and the associated cumulative incidence of fracture.

Results: Eighty-five patients administered GEJ met the inclusion criteria. After propensity score matching (1:5), 425 patients who were not administered GEJ were included in the control group. There were no significant differences about the baseline characteristics between the study and control groups. In the study group, GEJ improved the osteoporosis of the lumbar vertebrate and osteopenia of the hip joint but did not improve osteoporosis of the femoral neck. The cumulative rate of fracture between these two groups was not significantly different. Patients who took at least $600 \mathrm{GEJ}$ pills over the treatment period had a decreased risk for fracture at fracture-prone sites.

\section{Conclusion:}

The use of Guilu Erxian Jiao may improve osteoporosis of the lumbar vertebrate and osteopenia of the hip joint. In addition, it may help decrease fractures of the lumbar spine. Administering at least $600 \mathrm{GEJ}$ pills over the treatment period might result in a decreased risk for fracture at fracture-prone sites.

\section{Introduction}

Osteoporosis is a life threatening problem worldwide, and even in the highly developed United States, at least 10 million have osteoporosis [1]'[2]. People with osteoporosis experience hip fracture sooner, which is a fatal event in elderly individuals[3]. Thirty percent of patients die within one year after their first hip fracture[4]. Osteoporosis can be divided into two groups, primary osteoporosis and secondary osteoporosis. Primary osteoporosis affects postmenopausal women and men aged over 65 years. In addition, low body mass index, previous fragility fractures, a family history of fractures, the use of glucocorticoids, active cigarette smoking, and alcohol consumption are all risk factors for osteoporosis [5]. The treatments for osteoporosis fall into two categories, anabolic drugs that stimulate bone formation, and antiresorptive drugs that slow bone resorption. At least one year of treatment is required to achieve the goal of decreasing the fracture rate. It is suggested that bone mineral density (BMD) assessed with dual X-ray absorptiometry (DXA) should be performed every two years in osteoporosis patients [6]. 
Chinese herbal medicines (CHMs) have fewer side effects and are mainstream therapies in Asian countries [7]. In traditional Chinese medicine (TCM) theory, osteoporosis is believed to occur due to a deficiency in Shen (kidney) essence, which plays a critical role in nourishing the bone and strengthening the skeleton [8]. Guilu Erxian Jiao(GEJ),consisting of ginseng, lycium, Carapax testudinis and Cornu cervi, is a nourishing Chinese herbal medicine that is labeled to treat osteoporosis and is covered by the National Health Insurance system in Taiwan [9]. In an animal study, GEJ promoted osteoblastic differentiation marker ALP activity and increased the production of bone morphogenetic protein-2, which is a key regulator of bone formation and osteoblastic differentiation [10]. In a clinical trial, GEJ was found to increase the mean BMD of the lumbar spine and femoral neck in postmenopausal women after 12 weeks of intake [11].

This was a retrospective study of the effect of GEJ, performed at the Chang Gung Memorial Hospital $(\mathrm{CGMH})$. We evaluated the improvement of the T-score and the cumulative hip fracture rate among patients administered osteoporosis drugs and/or GEJ.

\section{Materials And Methods}

\section{Data source}

Data on the participants in this cohort study were retrieved from the CGMH database. We collected relevant data from January 1,2000, to December 31,2019. The CGMH database provides anonymized and encrypted data on patients for research purposes.

\section{Study population}

The inclusion criteria for our population cohort study were as follows: 1. DXA examination was performed at least twice between 2000 to 2019, and the interval between the two DXA examinations was at least three months and no more than two years (Fig. 1) and 2. The patients were over 18 years of age. The exclusion criteria were as follows: 1. patients who received GEJ treatment more than 90 days before the first DXA examination, 2. patients with incomplete data, and 3. patients with a T-score >-2.5.

Approval for the study was obtained from the Chang Gung Medical Foundation Institutional Review Board.

The background characteristics of the patients are described in Table 1. Comorbidities included cancer, cardiovascular disease, chronic kidney disease, chronic obstructive pulmonary disease, dementia, diabetes mellitus, depression, hypertension, Parkinson's disease, hyperparathyroidism, multiple myeloma, and rheumatic arthritis [12]. The medications administered for osteoporosis were bisphosphates, RANKL inhibitors, sex hormones, calcitriol, teriparatide, and steroids. Osteoporosis was defined as a T-score $\leq-2.5$ [13]. 
Table 1

Demographic data and selected comorbidities of the study subjects

\begin{tabular}{|c|c|c|c|c|c|}
\hline \multirow[t]{3}{*}{ Variables } & \multicolumn{2}{|c|}{ Control group } & \multicolumn{3}{|c|}{ Study group } \\
\hline & \multicolumn{2}{|c|}{$n=425$} & \multicolumn{2}{|l|}{$\mathrm{n}=85$} & \multirow[t]{2}{*}{ p-value } \\
\hline & mean & SD & mean & SD & \\
\hline Age at 1 st DXA(year) & 62.35 & 12.20 & 61.05 & 12.87 & 0.375 \\
\hline \multirow[t]{2}{*}{ Interval between the 1st and 2nd DXA } & 14.65 & 5.28 & 14.86 & 5.04 & 0.733 \\
\hline & $\mathrm{n}$ & $\%$ & $\mathrm{n}$ & $\%$ & \\
\hline \multicolumn{6}{|l|}{ Age } \\
\hline Age $<65$ & 280 & $65.88 \%$ & 56 & $65.88 \%$ & 1.000 \\
\hline Age $\geq 65$ & 145 & $34.12 \%$ & 29 & $34.12 \%$ & \\
\hline \multicolumn{6}{|l|}{ Gender } \\
\hline Male & 70 & $16.47 \%$ & 14 & $16.47 \%$ & 1.000 \\
\hline Female & 355 & $83.53 \%$ & 71 & $83.53 \%$ & \\
\hline \multicolumn{6}{|l|}{ Fracture before 1st DXA } \\
\hline Femoral neck fracture & 2 & $0.47 \%$ & 0 & $0.00 \%$ & 1.000 \\
\hline Lumbar vertebra fracture & 17 & $4.00 \%$ & 5 & $5.88 \%$ & 0.390 \\
\hline Thoracic vertebra fracture & 9 & $2.12 \%$ & 1 & $1.18 \%$ & 1.000 \\
\hline Radius ulna fracture & 7 & $1.65 \%$ & 1 & $1.18 \%$ & 1.000 \\
\hline Proximal humerus fracture & 3 & $0.71 \%$ & 0 & $0.00 \%$ & 1.000 \\
\hline \multicolumn{6}{|l|}{ Comorbidity } \\
\hline Cancer & 88 & $20.71 \%$ & 16 & $18.82 \%$ & 0.806 \\
\hline Cardiovascular disease & 57 & $13.41 \%$ & 16 & $18.82 \%$ & 0.258 \\
\hline Chronic kidney disease & 22 & $5.18 \%$ & 0 & $0.00 \%$ & 0.035 \\
\hline COPD & 80 & $18.82 \%$ & 13 & $15.29 \%$ & 0.538 \\
\hline Dementia & 6 & $1.41 \%$ & 3 & $3.53 \%$ & 0.177 \\
\hline Diabetes Mellitus & 59 & $13.88 \%$ & 6 & $7.06 \%$ & 0.123 \\
\hline Depression & 22 & $5.18 \%$ & 6 & $7.06 \%$ & 0.442 \\
\hline Hypertension & 121 & $28.47 \%$ & 23 & $27.06 \%$ & 0.895 \\
\hline
\end{tabular}




\begin{tabular}{|llllll|}
\hline Variables & \multicolumn{7}{l}{ Control group } & \multicolumn{2}{l|}{ Study group } & \\
\hline Parkinson's disease & 2 & $0.47 \%$ & 2 & $2.35 \%$ & 0.131 \\
\hline Hyperparathyroidism & 4 & $0.94 \%$ & 2 & $2.35 \%$ & 0.263 \\
\hline Multiple myeloma & 1 & $0.24 \%$ & 0 & $0.00 \%$ & 1.000 \\
\hline Rheumatic arthritis & 17 & $4.00 \%$ & 2 & $2.35 \%$ & 0.753 \\
\hline Medication for Osteoporosis & & & & & \\
\hline Bisphosphonates & 87 & $20.47 \%$ & 15 & $17.65 \%$ & 0.656 \\
\hline RANKL inhibitors & 81 & $19.06 \%$ & 15 & $17.65 \%$ & 0.879 \\
\hline Sex Hormones & 80 & $18.82 \%$ & 11 & $12.94 \%$ & 0.255 \\
\hline Calcitriol & 20 & $4.71 \%$ & 3 & $3.53 \%$ & 0.781 \\
\hline Teriparatide & 16 & $3.76 \%$ & 3 & $3.53 \%$ & 1.000 \\
\hline Steroid & 43 & $10.12 \%$ & 8 & $9.41 \%$ & 1.000 \\
\hline
\end{tabular}

\section{Study design}

Patients who received GEJ treatment and those who did not receive GEJ treatment were divided into the study group and control group, respectively, with 1:5 matching, which did not result in any significant differences in the basic values (Table 1).

\section{Assessment}

We assessed the T-score and cumulative rate of fracture to determine the effect of GEJ on osteoporosis patients.

\section{Statistical analysis}

The categorical variables are reported as numbers. The difference in proportions and T-scores were assessed using Student's t-test as appropriate. Cox's proportional hazard model was used to estimate hazard ratios (HRs) for the effect of GEJ on fracture rates. The difference in fracture occurrence between the two groups was estimated using the Kaplan-Meier method and a log-rank test. The database software SAS Enterprise Guide version 9.4 (SAS Institute Inc., Cary, NC, USA) was used for data processing and analysis, and $p<0.05$ was considered statistically significant.

\section{Results}


Two hundred eighteen patients administered GEJ and 19879 patients who were not administered GEJ met the inclusion criteria. After applying the exclusion criteria, 85 osteoporosis patients remained in the study group. We performed propensity score matching to enroll 425 osteoporosis patients in the control group. There was no significant difference between the two groups in terms of age, sex, interval between the two DXA assessments, fracture before the 1st DXA, comorbidity, or osteoporosis medication usage (Table 1). However, the proportion of osteoporosis medication use in the control group (67\%) was greater than that in the study group (55\%). The effect of anti-osteoporosis drugs is beyond doubt. As shown in Table 2, the hip condition of patients in the control group was worse than that of patients in the study group $(p=0.036)$.

Table 2

T-scores of the lumbar spine, hip, and femoral neck before and after examination in the study and control groups of osteoporosis patients

\begin{tabular}{|lccccc|}
\hline \multicolumn{5}{|c|}{ Control group } & \multicolumn{2}{c|}{ Study group } \\
\hline & $\mathrm{n}=425$ & $\mathrm{n}=85$ & & $\mathrm{p}$-value \\
\hline & mean & SD & mean & SD & \\
\hline Lumbar & & & & & \\
\hline T-score before & -2.77 & 1.08 & -2.61 & 1.03 & 0.235 \\
\hline T-score after & -2.56 & 1.13 & -2.42 & 1.04 & 0.298 \\
\hline L Hip & & & & & \\
\hline T-score before & -2.51 & 0.81 & -2.30 & 0.84 & 0.036 \\
\hline T-score after & -2.43 & 0.86 & -2.19 & 0.89 & 0.024 \\
\hline L_Fneck & & & & & \\
\hline T-score before & -3.08 & 0.86 & -2.88 & 1.14 & 0.124 \\
\hline T-score after & -2.98 & 0.88 & -2.78 & 0.83 & 0.057 \\
\hline L, left; F, femoral. & & & & \\
\hline p < 0.05 were bold. & & & & \\
\hline
\end{tabular}

In these two groups, there was a significant difference in the improvements in T scores at the lumbar spine and hip joint. However, there was no significant improvement in the T score at the left femoral neck (Table 3). As shown in Table 4, which shows the number of fractures at each joint, in the study group, the number of fractures of the lumbar joint after the 1st DXA was significantly lower than that in the control group. 
Table 3

T-score before and after GEJ and intragroup comparison

\begin{tabular}{|lllllll|}
\hline & \multicolumn{2}{c}{ Control group } & \multicolumn{3}{c|}{ Study group } \\
\cline { 2 - 7 } & $\mathbf{n = 4 2 5}$ & p-value & $\mathbf{n = 8 5}$ & p-value \\
\hline & mean & sd & & mean & sd & \\
\hline Lumbar & & & & & & \\
\hline T-score before vs T-score after & 0.21 & 0.58 & $<\mathbf{0 . 0 0 0 1}$ & 0.19 & 0.46 & $\mathbf{0 . 0 0 0 2}$ \\
\hline L_Hip & & & & & & \\
\hline T-score before vs T-score after & 0.08 & 0.39 & $<0.0001$ & 0.11 & 0.39 & $\mathbf{0 . 0 1 1 7}$ \\
\hline L_Femoral neck & & & & & & \\
\hline T-score before vs T-score after & 0.10 & 0.56 & $\mathbf{0 . 0 0 0 2}$ & 0.08 & 1.09 & 0.4839 \\
\hline p $<0.05$ were bold. & & & & & & \\
\hline
\end{tabular}

Table 4

Fracture rate after the first DXA in the study group and control group.

\begin{tabular}{|lccccc|}
\hline Fracture after 1st DXA(new case) & \multicolumn{3}{l}{ Control group } & \multicolumn{3}{l|}{ Study group } \\
& $\mathrm{n}$ & $\%$ & $\mathrm{n}$ & $\%$ & $\mathrm{p}$-value \\
\hline Femoral neck fracture & 3 & $0.71 \%$ & 0 & $0.00 \%$ & 1.000 \\
\hline Lumbar vertebrae fracture & 58 & $13.65 \%$ & 4 & $4.71 \%$ & $\mathbf{0 . 0 3 4}$ \\
\hline Thoracic vertebrae fracture & 20 & $4.71 \%$ & 5 & $5.88 \%$ & 0.588 \\
\hline Radius ulna fracture & 14 & $3.29 \%$ & 3 & $3.53 \%$ & 1.000 \\
\hline Proximal humerus fracture & 3 & $0.71 \%$ & 1 & $1.18 \%$ & 0.519 \\
\hline $\mathrm{p}<0.05$ were bold. & & & & & \\
\hline
\end{tabular}

Table 5 shows the risk of fracture occurrence in the two groups. There was no significant difference between the groups except for in patients taking bisphosphonate $s(p=0.0012)$. Bisphosphonate-treated patients experienced more fractures before the 1st DXA than those who were not administered bisphosphonates, and the difference was significantly different $(p<0.001$; Table 6$)$. 
Table 5

The fracture rate after treatment with medications and/or Guilu Erxian Jiao.

\begin{tabular}{|llll|}
\hline \multicolumn{4}{|c|}{ Univariate cox } \\
\hline & HR & $95 \% \mathrm{Cl}$ & p-value \\
\hline Group & & & \\
\hline Guilu Erxian Jiao Treatment (A) & 0.70 & $0.33-1.46$ & 0.338 \\
\hline No Guilu Erxian Treatment (B) & ref & & \\
\hline Medication for Osteoporosis & & & \\
\hline Bisphosphates & 1.94 & $1.16-3.24$ & 0.012 \\
\hline RANKL inhibitors & 1.34 & $0.75-2.38$ & 0.323 \\
\hline Sex hormones & 0.84 & $0.44-1.61$ & 0.603 \\
\hline Calcitriol & 0.61 & $0.15-2.48$ & 0.486 \\
\hline Teriparatide & 2.26 & $0.91-5.61$ & 0.08 \\
\hline Steroid & 1.16 & $0.56-2.43$ & 0.689 \\
\hline
\end{tabular}

Table 6

The fracture rate before the first DXA with or without bisphosphonates

\section{Bisphosphonates}

\begin{tabular}{|c|c|c|c|c|c|}
\hline & \multicolumn{2}{|c|}{ No Bisphosphonates } & \multicolumn{2}{|c|}{ Bisphosphonates } & \multirow[t]{2}{*}{ P-value } \\
\hline & $\mathrm{N}$ & $\%$ & $\mathrm{~N}$ & $\%$ & \\
\hline No fracture before the 1st DXA & 390 & 95.59 & 82 & 80.39 & $<0.0001$ \\
\hline Fracture before the 1st DXA & 18 & 4.41 & 20 & 19.61 & \\
\hline
\end{tabular}

The Kaplan-Meier survival curve and log-rank test results also revealed no statistically significant difference in the fracture-free survival rate between the two groups during the five-year follow-up period. Those receiving GEJ did not have a significantly lower fracture incidence rate than those not receiving GEJ ( $p=0.335$; Fig. 2). We subdivided the control group into the medication for osteoporosis + non-GEJ group and the nonmedication for osteoporosis + non-GEJ group and subdivided the study group into the medication for osteoporosis + GEJ group and the nonmedication for osteoporosis + GEJ group.

The Kaplan-Meier analysis of the difference between these four groups also showed no significant difference in the fracture incidence rate among them (Fig. 3). 
The receiver operating characteristic curve (ROC curve) analysis indicated that a total number GEJ pills of 600 was an ideal discrimination point (Fig. 4). There was a significant difference in fracture development rates between the high total dose GEJ group and the low total dose GEJ group ( $p=0.0039$; Fig. 5).

\section{Discussion}

The reduction of osteoporosis prevalence and incidence is critical due to the increase in the aging population in developing countries [14]'[15]. Many studies have emphasized the importance of traditional Chinese medicine for treating osteoporosis and preventing fractures [9],[16, 17]. Among these medicines, GEJ has often been prescribed [18]'[19]. In a clinical study, improvement in the BMD of the lumbar spine in postmenopausal women (mean age 54.8 [20][21]) after three months of GEJ treatment was observed [11]. Unfortunately, most of the data analyzed in previous studies were obtained through the $\mathrm{NHI}$ database; thus, there is a lack of follow-up reports. Therefore, we set the first inclusion criterion to select osteoporosis patients who had undergone two DXA examinations to observe the effect of GEJ.

GEJ was the most prescribed formula at the $\mathrm{CGMH}$, and the number of osteoporosis patients examined by DXA twice exceeded 40,000. However, only 218 patients were both administered GEJ and evaluated by DXA twice. The reason for this small number might be that some patients prescribed GEJ at the CGMH received DXA examination at other hospitals, leading to the loss of clinical data. While the data were anonymized and delinked, the DXA reporting forms and contents differed depending on the radiologist performing the evaluation. This also reduced the number of included patients. The results of a previous Taiwan NHI database study demonstrated that TCM use can decrease the rate of osteoporotic fracture [9]. In our study, GEJ was the main prescribed formula. However, our study results were not in agreement with the results of the aforementioned study. This might be due to the large difference in the total number of GEJ patients enrolled in our retrospective study.

Although we did not observe the expected results, we found that GEJ might improve osteoporosis of the lumbar spine. The number of fractures of the lumbar spine was significantly different $(p=0.034$, Table 4$)$. According to the results of previous studies, ginseng, a component of GEJ, can inhibit skeletal muscle atrophy, enhance muscle performance and improve blood circulation[22-25]. Another Chinese herb in the GEJ formula, lycium, can also improve muscle endurance [26]. These functions might help prevent elderly individuals from falling. This may be the reason the proportion of lumbar spine fractures in the study group was lower than that in the control group. Our database did not include the number of falls experienced by patients, which may be important data to analyze in our future studies. Although osteopenia at the hip joint was present in the study group, there was significant improvement in the patients' condition. This indicates that GEJ may play an important role in the prevention of hip fractures [4] [27]. A previous animal study also showed that GEJ can be used to treat resorptive diseases, such as osteoporosis, by inhibiting osteoclast resorption [28]. This result is compatible with the results of our study. We demonstrated that patients administered a high GEJ dose of at least 600 pills in total had a lower incidence of fractures. This result may guide future studies. 
This study has several limitations. First, the sample size of patients who received GEJ treatment was small, as DXA data could not be obtained from other hospitals. Second, investigations into the impact of ethical, psychological, and cultural factors were limited due to the nature of the database, which restricts investigations into differences between rural and urban areas.

\section{Conclusion}

This is the first cohort study to investigate the effect of GEJ on osteoporosis risk and the fracture rates among osteoporosis patients. The results of this study suggest that GEJ might improve osteoporosis of the lumbar spine and decrease the fracture rate of the lumbar spine. Patients who take at least $600 \mathrm{GEJ}$ pills over the treatment period might have a decreased risk for fracture at fracture-prone sites.

\section{Abbreviations}

GEG

Guilu Erxian Jiao; CGMH:Chang Gung Memorial Hospital; BMD:bone mineral density; DXA:dual X-ray absorptiometry; CHMs:Chinese herbal medicines; TCM:traditional Chinese medicine; HRs:hazard ratios; ROC curve:receiver operating characteristic curve.

\section{Declarations}

\section{Acknowledgments}

We are very grateful to the people assisting with the statistical analyses in the study in Chang Gung Memorial Hospital Center for Big Data Analytics and Statistics who have provided valuable information about study design. monitoring, data analysis.

\section{Funding}

This work was supported by a grant from the Chang Gung Memorial Hospital (CORPG1H0051). The authors thank Shu-Ting, Ganc and Hui Ming, Chen for their assistance with the statistical analyses, and wish to acknowledge the support of the Maintenance Project of the Center for Big Data Analytics and Statistics (Grant CLRPG3D0048) at the Chang Gung Memorial Hospital for help with the study design. monitoring, data analysis and interpretation. This study is based in part on data from the Chang Gung Research Database provided by the Chang Gung Memorial Hospital. The interpretation and conclusions contained herein do not represent the position of the Chang Gung Memorial Hospital.

\section{Author Contribution Statement}

Yuan-Xin Lai and Tsung-Hsien Yang designed the experiments, performed the data analysis and statistical analysis, and drafted the manuscript. Chu-yao Tseng participated in the study design and 
helped with the data analysis. Pai-Wei Lee Yang helped with the data analysis. Huang-Yu and Sien-Hung Yang provided professional comments on this work. All authors read and approved the final manuscript.

\section{Author Disclosure Statement}

No competing financial interests exist

\section{Availability of data and materials}

Please contact author for data requests.

\section{Ethics approval and consent to participate}

Prior to commencement of the study, ethical approval (201800780B0C501) (Additional file 1) was obtained from the Chang Gung Medical Foundation Institutional Review Board.

\section{Consent to publish}

The authors give full consent to publish.

\section{References}

1. Srivastava M, Deal C. Osteoporosis in elderly: prevention and treatment. Clinics in Geriatric Medicine. 2002;18:529-55. doi: https://doi.org/10.1016/S0749-0690(02)00022-8.

2. Osteoporosis prevention, diagnosis, and therapy. Jama. 2001;285:785-95. doi: 10.1001/jama.285.6.785.

3. Lewiecki EM, Wright NC, Curtis JR, Siris E, Gagel RF, Saag KG, et al. Hip fracture trends in the United States, 2002 to 2015. Osteoporos Int. 2018;29:717-22. doi: 10.1007/s00198-017-4345-0.

4. Lee $\mathrm{SH}$, Chen IJ, Li YH, Fan Chiang $\mathrm{CY}$, Chang $\mathrm{CH}$, Hsieh $\mathrm{PH}$. Incidence of second hip fractures and associated mortality in Taiwan: A nationwide population-based study of 95,484 patients during 2006-2010. Acta Orthop Traumatol Turc. 2016;50:437-42. doi: 10.1016/j.aott.2016.06.008.

5. Lane NE. Epidemiology, etiology, and diagnosis of osteoporosis. Am J Obstet Gynecol. 2006;194:S311. doi: 10.1016/j.ajog.2005.08.047.

6. Rachner TD, Khosla S, Hofbauer LC. Osteoporosis: now and the future. The Lancet. 2011;377:127687. doi: 10.1016/s0140-6736(10)62349-5.

7. Zhou X, Li CG, Chang D, Bensoussan A. Current Status and Major Challenges to the Safety and Efficacy Presented by Chinese Herbal Medicine. Medicines (Basel). 2019;6. doi: 10.3390/medicines6010014.

8. Liu Y, Liu JP, Xia Y. Chinese herbal medicines for treating osteoporosis. Cochrane Database Syst Rev. 2014:CD005467. doi: 10.1002/14651858.CD005467.pub2. 
9. Wang YC, Chiang JH, Hsu HC, Tsai $\mathrm{CH}$. Decreased fracture incidence with traditional Chinese medicine therapy in patients with osteoporosis: a nationwide population-based cohort study. BMC Complement Altern Med. 2019;19:42. doi: 10.1186/s12906-019-2446-3.

10. Wu MH, Lee TH, Lee HP, Li TM, Lee IT, Shieh PC, et al. Kuei-Lu-Er-Xian-Jiao extract enhances BMP-2 production in osteoblasts. Biomedicine (Taipei). 2017;7:2. doi: 10.1051/bmdcn/2017070102.

11. Li R-S, Shyu J-Y, Sun S-Y, Ho F-M, Wang S-H. The Effect of Guilu Erxian Decoction on Bone Mineral Density in Postmenopausal Women Evaluated Using Dual Energy X-ray Absorptiometry and Quantitative Ultrasound. C J Radiologic Tech. 2007;31:147-53. doi: 10.30045/cjrt.200712.0009.

12. Hu RH, Hsiao FY, Chen LJ, Huang PT, Hsu WW. Increasing age- and gender-specific burden and complexity of multimorbidity in Taiwan, 2003-2013: a cross-sectional study based on nationwide claims data. BMJ Open. 2019;9:e028333. doi: 10.1136/bmjopen-2018-028333.

13. Sozen T, Ozisik L, Basaran NC. An overview and management of osteoporosis. Eur J Rheumatol. 2017;4:46-56. doi: 10.5152/eurjrheum.2016.048.

14. Aziziyeh R, Amin M, Habib M, Garcia Perlaza J, Szafranski K, McTavish RK, et al. The burden of osteoporosis in four Latin American countries: Brazil, Mexico, Colombia, and Argentina. J Med Econ. 2019;22:638-44. doi: 10.1080/13696998.2019.1590843.

15. Burge R, Dawson-Hughes B, Solomon DH, Wong JB, King A, Tosteson A. Incidence and economic burden of osteoporosis-related fractures in the United States, 2005-2025. J Bone Miner Res. 2007;22:465-75. doi: 10.1359/jbmr.061113.

16. Jin YX, Wu P, Mao YF, Wang B, Zhang JF, Chen WL, et al. Chinese Herbal Medicine for Osteoporosis: A Meta-analysis of Randomized Controlled Trials. J Clin Densitom. 2017;20:516-25. doi: 10.1016/j.jocd.2017.07.003.

17. Lee H, Kim MH, Choi Y, Yang WM. Ameliorative effects of Osteo-F, a newly developed herbal formula, on osteoporosis via activation of bone formation. J Ethnopharmacol. 2021;268:113590. doi: 10.1016/j.jep.2020.113590.

18. Shih WT, Yang YH, Chen PC. Prescription patterns of chinese herbal products for osteoporosis in taiwan: a population-based study. Evid Based Complement Alternat Med. 2012;2012:752837. doi: $10.1155 / 2012 / 752837$.

19. Zhang ND, Han T, Huang BK, Rahman K, Jiang YP, Xu HT, et al. Traditional Chinese medicine formulas for the treatment of osteoporosis: Implication for antiosteoporotic drug discovery. J Ethnopharmacol. 2016;189:61-80. doi: 10.1016/j.jep.2016.05.025.

20. Shen TY, Strong C, Yu T. Age at menopause and mortality in Taiwan: A cohort analysis. Maturitas. 2020;136:42-8. doi: 10.1016/j.maturitas.2020.04.008.

21. Wang M, Gong WW, Hu RY, Wang H, Guo Y, Bian Z, et al. Age at natural menopause and associated factors in adult women: Findings from the China Kadoorie Biobank study in Zhejiang rural area. PLoS One. 2018;13:e0195658. doi: 10.1371/journal.pone.0195658.

22. Seok YM, Yoo JM, Nam Y, Kim J, Kim JS, Son JH, et al. Mountain ginseng inhibits skeletal muscle atrophy by decreasing muscle RING fi nger protein-1 and atrogin1 through forkhead box 03 in L6 
myotubes. J Ethnopharmacol. 2021;270:113557. doi: 10.1016/j.jep.2020.113557.

23. In-Ho C, Byung-Woo K, Yun-Jae P, Han-Joo L, Sok P, Namju L. Ginseng berry extract increases nitric oxide level in vascular endothelial cells and improves cGMP expression and blood circulation in muscle cells. J Exerc Nutrition Biochem. 2018;22:6-13. doi: 10.20463/jenb.2018.0018.

24. Xin G-j, Zhao Y-w, Li L-m, Jia F-f, Han X, Li L, et al. Mechanism of 'Invigorating Qi and Promoting Blood Circulation' Drug Pair Ginseng-Danshen on Treatment of Ischemic Heart Disease Based on Network Pharmacology. Chinese Journal of Integrative Medicine. 2021;27:440-5. doi: 10.1007/s11655-021-2859-y.

25. Sellami M, Slimeni O, Pokrywka A, Kuvacic G, L DH, Milic M, et al. Herbal medicine for sports: a review. J Int Soc Sports Nutr. 2018;15:14. doi: 10.1186/s12970-018-0218-y.

26. Meng J, Lv Z, Sun C, Qiao X, Chen C. An extract of Lycium barbarum mimics exercise to improve muscle endurance through increasing type lla oxidative muscle fibers by activating ERRgamma. FASEB J. 2020;34:11460-73. doi: 10.1096/fj.202000136R.

27. Bouxsein ML, Eastell R, Lui LY, Wu LA, de Papp AE, Grauer A, et al. Change in Bone Density and Reduction in Fracture Risk: A Meta-Regression of Published Trials. J Bone Miner Res. 2019;34:63242. doi: 10.1002/jbmr.3641.

28. Yun Z, Cuiping M, Weimin Y, Xiaoxiang Z, editors. Application of cell engineering of herbal Medicine treating bone resorption of osteoclasts. 2005 IEEE Engineering in Medicine and Biology 27th Annual Conference; 2005 17-18 Jan. 2006.

\section{Figures}


2000-2019

Patients who received at least two DXA

examinations were included

$(n=44,062)$

The interval between DXA examinations was at least three months and no more than two years $(\mathrm{n}=21385)$

Exclude:

- T score $>-2.5 \mathrm{D}(\mathrm{n}=128)$

- Incomplete information ( $n=5)$

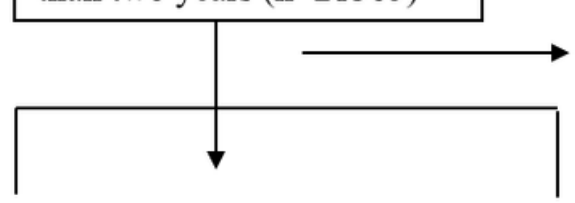

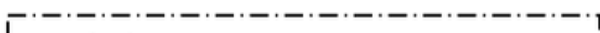
Exclude:

- Used Guilu Erxian Jiao before 90

day of DXA $(n=429)$

$\cdot$ Age $<18(\mathrm{n}=856)$

-No data $(\mathrm{n}=3)$

Guilu Erxian Jiao administered during follow-up period $(n=218)$
No Guilu Erxian Jiao administered during follow-up period $(\mathrm{n}=19879)$

\section{Figure 1}

Flowchart of the selection and follow-up of study subjects 


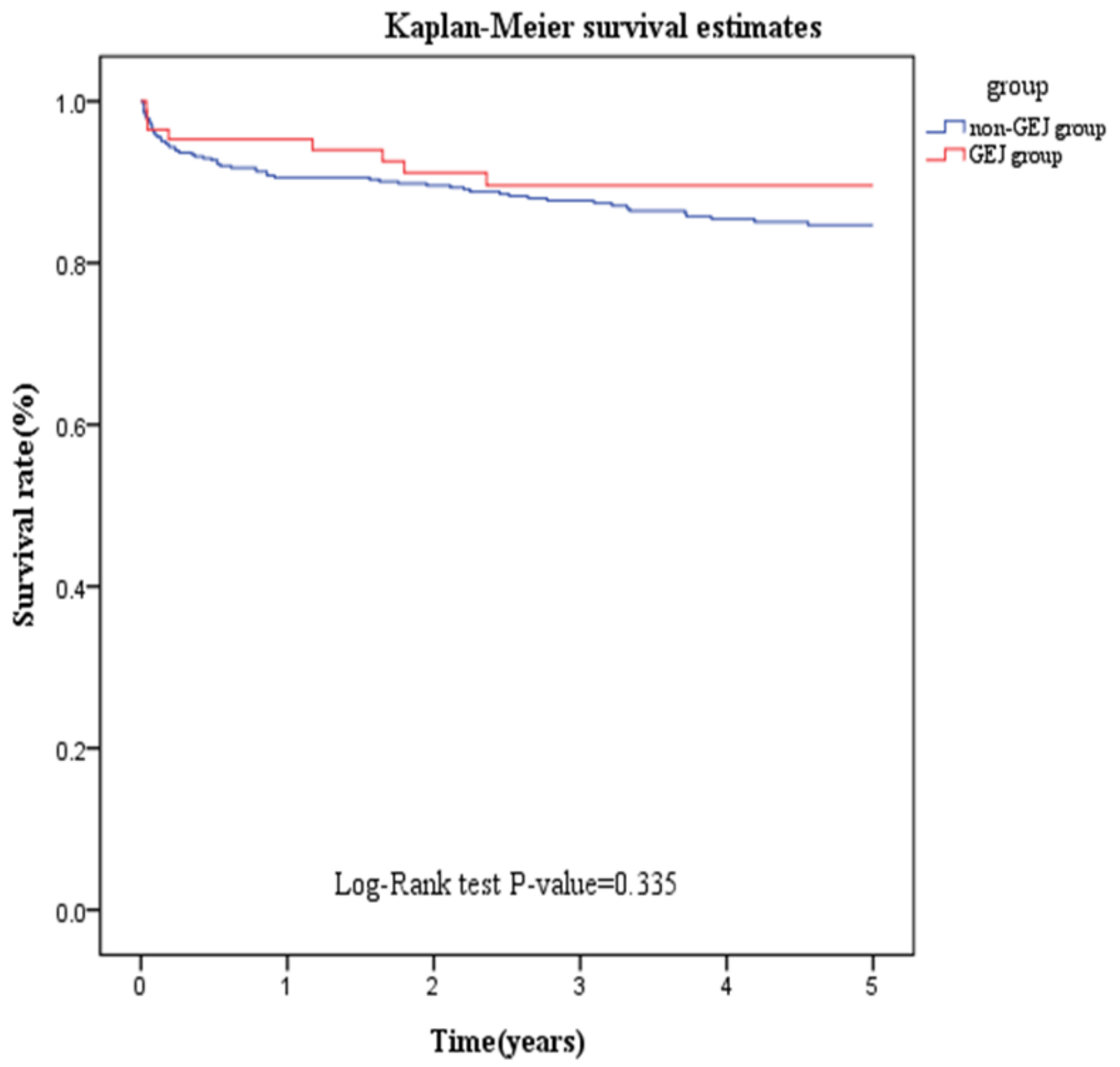

Figure 2

Comparison of Kaplan-Meier curves of the difference in fracture rates between those administered Guilu Erxian Jiao and those not administered Guilu Erxian Jiao. GEJ, Guilu Erxian Jiao. 


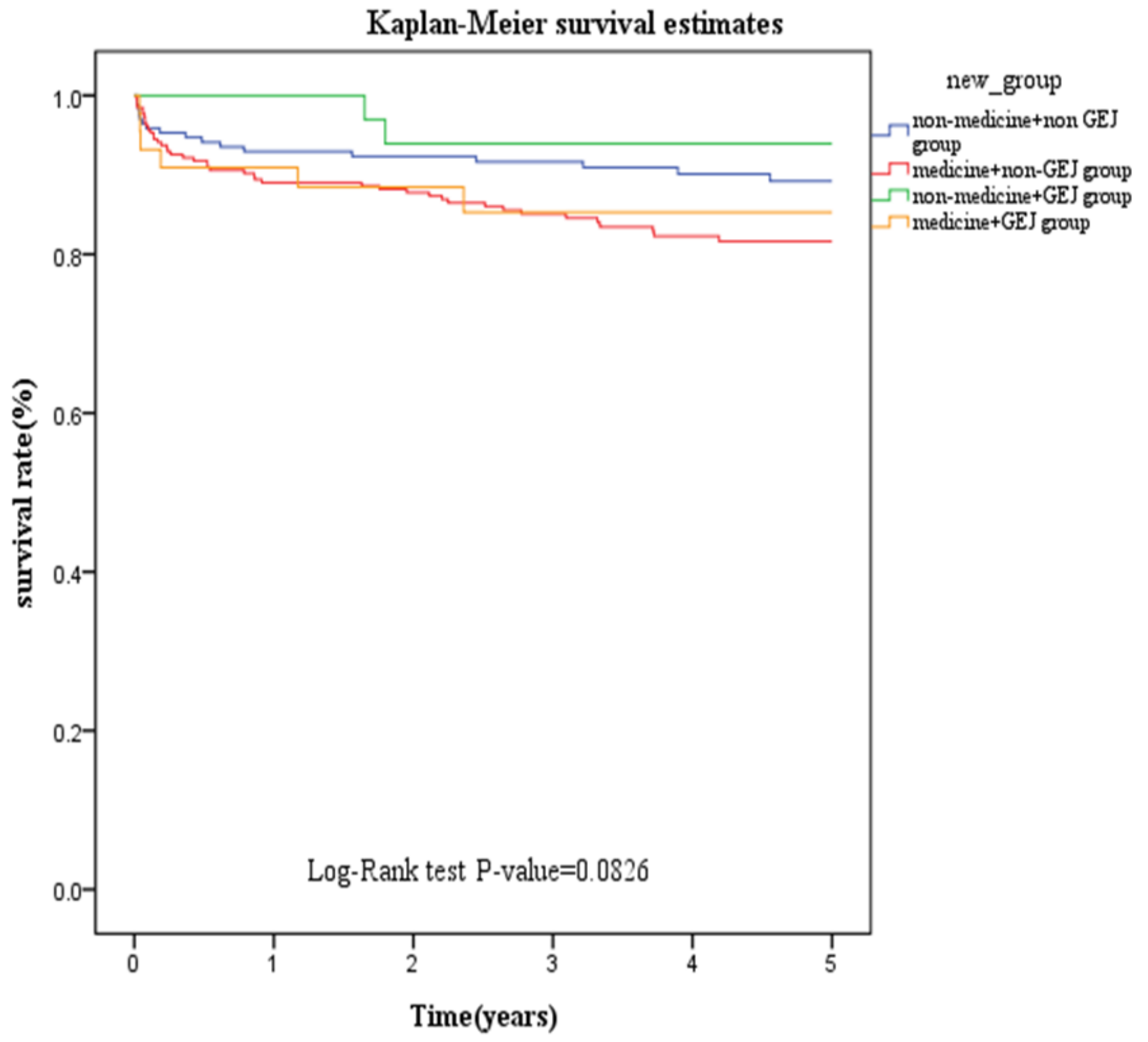

Figure 3

Comparison of Kaplan Meier curves of fracture onset among the four groups. GEJ, Guilu Erxian Jiao. 


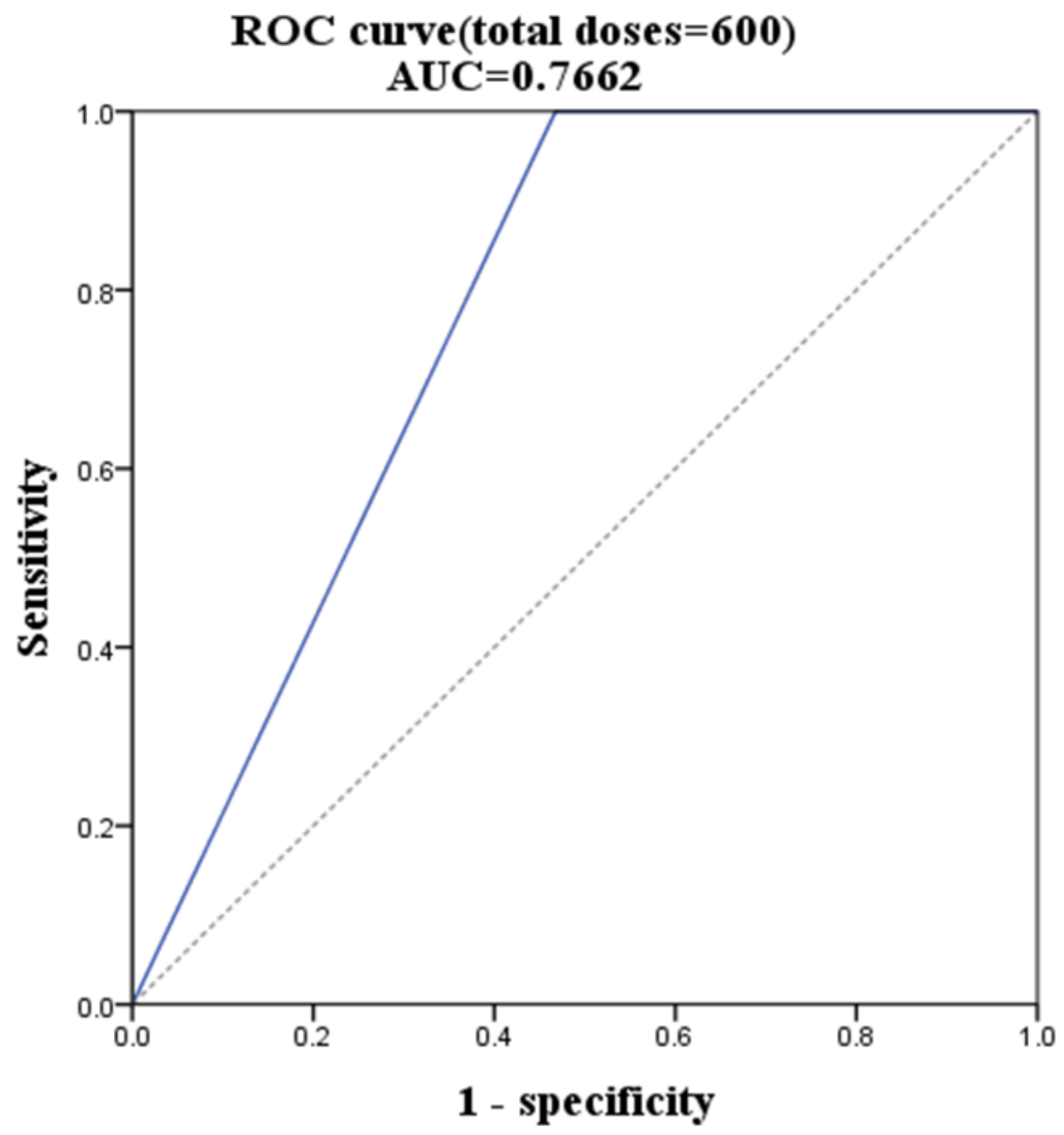

Figure 4

ROC curves according to the total dose of Guilu Erxian Jiao administered (dose multiplied by number of days of treatment) 


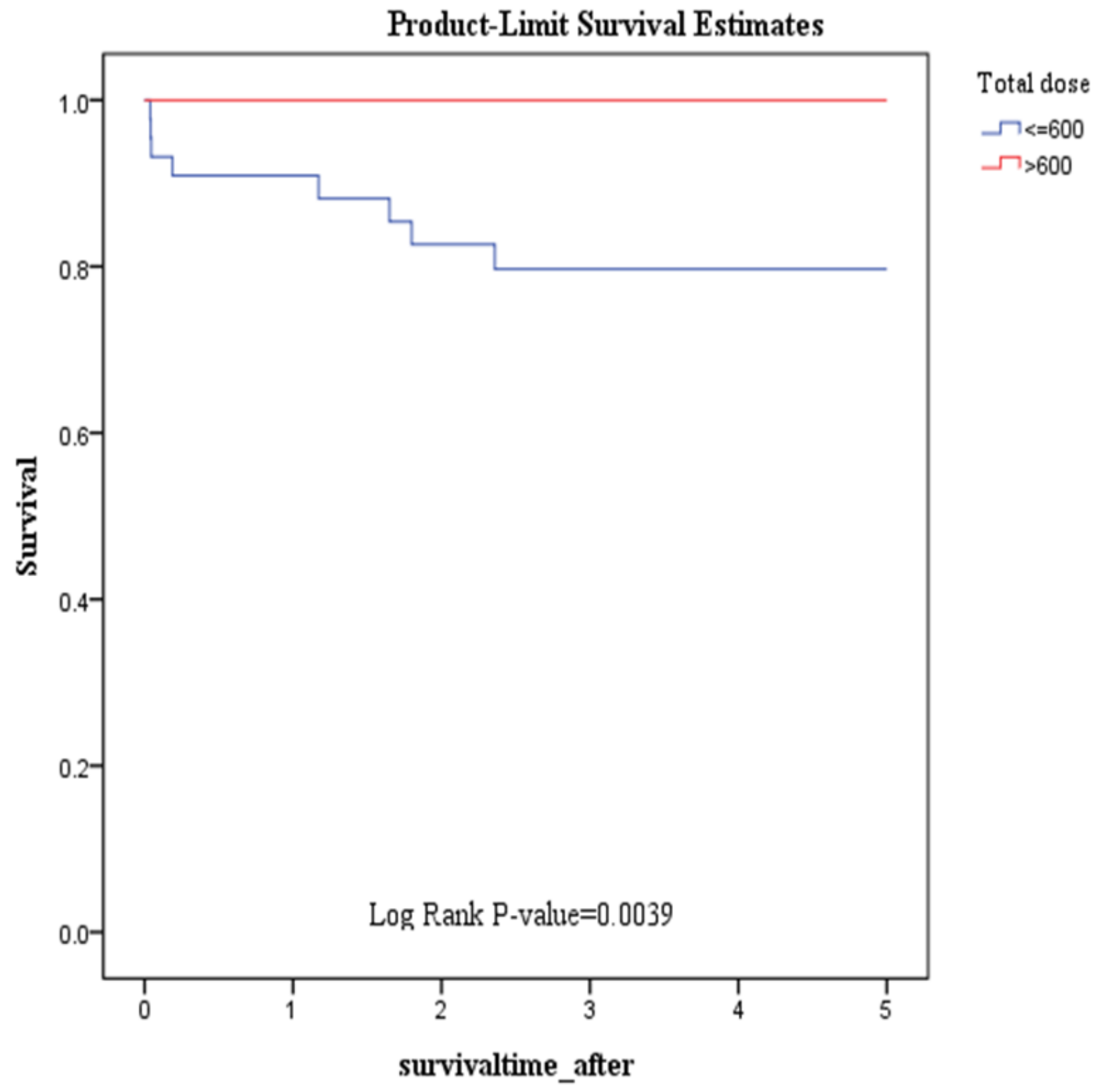

Figure 5

Kaplan-Meier curve of the difference in fracture development rates between those administered the high total dose of Guilu Erxian Jiao (>600 pills) and those administered the low total dose of Guilu Erxian Jiao (<=600 pills). 